\title{
Expression level of ECT2 proto-oncogene correlates with prognosis in glioma patients
}

\author{
MASAKAZU SANO $^{1}$, NOBUYUKI GENKAI ${ }^{1}$, NAOKI YAJIMA ${ }^{1}$, NAOTO TSUCHIYA ${ }^{1}$, \\ JUNPEI HOMMA ${ }^{1}$, RYUICHI TANAKA ${ }^{1}$, TORU MIKI ${ }^{2}$ and RYUYA YAMANAKA ${ }^{1,3}$ \\ ${ }^{1}$ Department of Neurosurgery, Brain Research Institute, Niigata University, 1-757 Asahimachi-dori, \\ Niigata 951-8585, Japan; ${ }^{2}$ Molecular Tumor Biology Section, Laboratory of Cell Biology, \\ National Cancer Institute, NIH Bldg. 37, Rm. 2144, 37 Convent Drive, Bethesda, MD 20892-4256, USA
}

Received May 31, 2006; Accepted June 26, 2006

\begin{abstract}
The ECT2 (epithelial cell transforming sequence 2) proto-oncogene encodes a guanine nucleotide exchange factor for Rho GTPases, and regulates cytokinesis. ECT2 plays a critical role in Rho activation during cytokinesis, and thus may play a role in the pathogenesis of glioma. In this study, we investigated relationships between ECT2 expression, tumor histology, and prognosis in glioma patients. ECT2 mRNA expression was examined using quantitative real-time PCR, while its protein expression was examined by immunohistochemistry of 54 glioma tissue samples. Expressions of ECT2 mRNA and protein were markedly increased in highgrade gliomas compared to low-grade gliomas. Patients in whom expression of ECT2 mRNA and protein in tumor tissues was the lowest survived longer than patients who had higher expression. In vitro, ECT2 siRNA inhibited glioma cell proliferation and invasion. These data suggest that increased expression of ECT2 contribute to promotion of tumor invasiveness and progression, implying that evaluation of ECT2 expression is a prognostic marker for glioma patients.
\end{abstract}

\section{Introduction}

Glioblastomas are ordinary solid tumors with poor prognosis. Despite recent advances in cancer diagnostic methodologies and treatments, prognosis of glioblastomas has not considerably improved worldwide $(1,2)$. Poor prognosis is attributable to difficulties of early detection and a high recurrence rate during post-initial treatment observation

Correspondence to: Dr Ryuya Yamanaka, ${ }^{3}$ Present address: Research Center of Innovative Cancer Therapy, Kurume University School of Medicine, Asahimachi 67, Kurume, Fukuoka 830-0011, Japan

E-mail: ryaman@med.kurume-u.ac.jp

Abbreviations: ECT2, epithelial cell transforming gene 2; MEM, minimum essential medium; PBS, phosphate buffered saline

Key words: epithelial cell transforming gene 2, glioma, siRNA periods. This is at least believed to be partly due to the lack of reliable tumor markers, and molecular targets against glioblastomas.

Epithelial cell transforming gene 2 (ECT2) proto-oncogene was originally isolated from epithelial cells as a cDNA clone, conferring transforming activity to fibroblasts, using an expression cloning strategy (3). ECT2 transfectants exhibit anchorage-independent cell growth, and efficient tumor formation in nude mice. The ECT2 cDNA encodes the Cterminal, half of the full-length protein containing Dblhomology (DH) and pleckstrin homology (PH) domains, which are found in a number of molecules involved in regulation of the Rho family GTPases $(4,5)$. The N-terminal half of ECT2 contains tandem BRCA1-C terminal (BRCT) repeats, that associate with proteins involved in DNA repair and cell cycle checkpoint responses $(4,5)$. ECT2 catalyzes guanine nucleotide exchange on RhoA, Rac1, and Cdc42 in vitro (4). The Rho family of small GTPases represented by RhoA, Rac1, and Cdc42, functions as molecular switches of diverse biological functions such as cytoplasmic actin reorganization, cell motility, cell scattering, cytokinesis, lymphocyte coagulation, smooth muscle contraction, and superoxide generation through induction of specific types of actin cytoskeleton, and local regulation of microtubule dynamics (6-8). The GTPbound form of Rho proteins is active, whereas the GDP-bound form is inactive. Activation of Rho proteins is promoted by guanine nucleotide exchange factors (GEFs), which catalyze replacement of bound GDP by GTP. The GTP-bound form of Rho proteins can specifically interact with effectors or targets, and transmits signals to downstream molecules. Rho proteins are inactivated through hydrolysis of bound GTP to GDP by intrinsic GTPase activity, assisted by GTPase activating proteins.

ECT2 is phosphorylated in the $\mathrm{G} 2$ and $M$ phases of the cell cycle by Cyclin B/Cyclin-dependent kinase 1, and is dephosphorylated before cytokinesis $(9,10)$. In the $M$ phase, ECT2 is localized in the mitotic spindles. Cell-cycle regulatorrelated domains of ECT2 play essential roles in the regulation of cytokinesis $(4,5,11)$. During cytokinesis, ECT2 is localized on the mid-body. Inhibition of ECT2 function, either by microinjection of affinity-purified anti-ECT2 antibodies or by expression of a dominant-negative form of ECT2 (ECT2-N), 

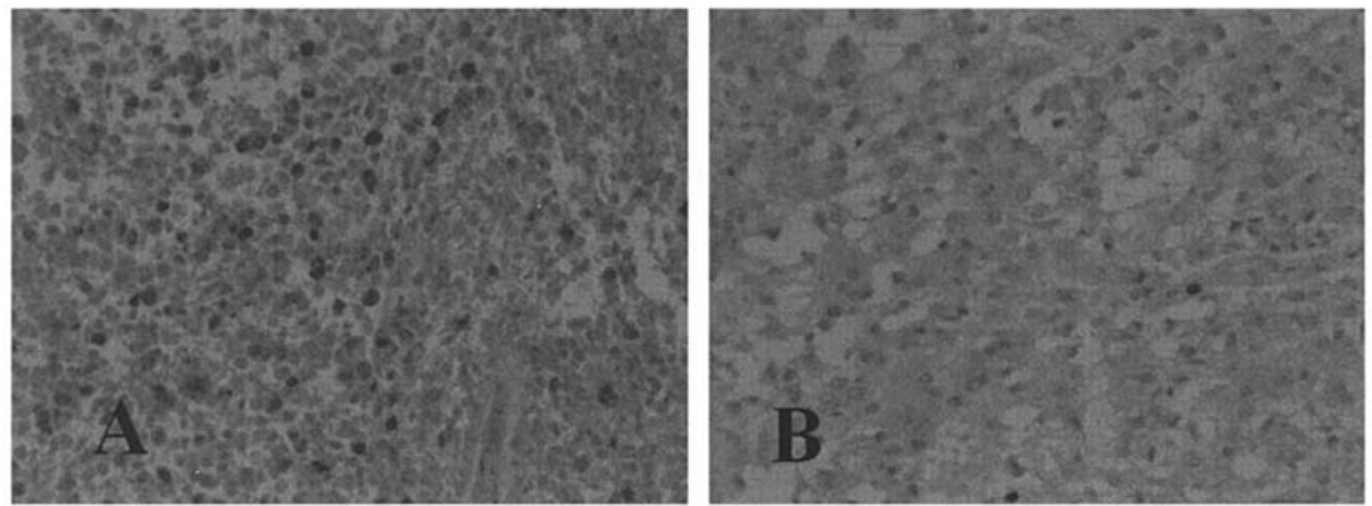

Figure 1. Representative immunohistochemical staining patterns with anti-ECT2 antibody. (A) Glioblastoma classified as showing high ECT2 expression. (B) Astrocytoma classified as showing low ECT2 expression. Magnification, x200.

strongly inhibits cytokinesis. The Drosophila gene Pebble, whose mutation renders cells defective in cytokinesis during embryogenesis, encodes an ECT2-related protein (12). In Drosophila cells, the Pavarotti/RacGAP50C complex accumulates at the central spindle, and association of Pebble with the complex activates the Rho signaling pathway to initiate cytokinesis (13). ECT2 was shown to play a critical role in Rho activation during cytokinesis, as dominant negative expression of ECT2 efficiently inhibited elevation of active Rho during cytokinesis (14). The critical cytokinesis regulator that is regulated by $\mathrm{Cdk} 1 /$ cyclin B may be ECT2, as suggested by genetic work in Drosophila (15). Therefore, ECT2 regulates cytokinesis from flies to humans. Removal of the central $\mathrm{S}$ domain containing nuclear localization signals stimulates transforming activity of ECT2 (5). The N-terminal domain of ECT2 associates with the C-terminal domain, and deletion of the N-terminal half of ECT2 stimulates its transforming activity (5). ECT2 activates several Rho-regulated signaling pathways, leading to malignant transformation (5).

In this study, we investigated expression of ECT2 in 54 glioma tissue specimens of different grades, and identified whether increased expression of ECT2 correlated with patients' prognosis.

\section{Materials and methods}

Cell lines and culture. All glioma cell lines were cultured in MEM (Nissui Pharmaceutical Inc., Tokyo, Japan) supplemented with 10\% FBS (fetal bovine serum). The T98G, GI-1 and U251 cell lines were purchased from Cell Bank, RIKEN BioResource Center (Tsukuba, Japan).

Patient characteristics and tissue samples. A total of 54 glioma tissue samples were investigated that were surgically removed at the Department of Neurosurgery, Brain Research Institute, Niigata University. Tumor tissue and normal brain tissue specimens were dissected, and immediately frozen at $-80^{\circ} \mathrm{C}$. Patient data are shown in Table I. Following surgical resection of tumors, patients with high-grade glioma started a course of external beam radiation therapy (standard dose, $60 \mathrm{~Gy}$ to the tumor) and nitrosourea-based chemotherapy during the course of the disease. Survival was determined from the date of diagnosis to death or last visit. Diagnoses were based on the World Health Organization (WHO) criteria (16). This study was approved by the Ethics Committee of Niigata University. Informed consent was obtained from all patients.

Immunohistochemistry. Sections ( $5 \mu \mathrm{m})$ from formalin-fixed, paraffin-embedded tissue specimens were deparaffinized in xylene, and dehydrated in a graded series of ethanol and PBS. Antigen retrieval was done by incubation at $121^{\circ} \mathrm{C}$ for $10 \mathrm{~min}$ in $10 \mathrm{mM}$ sodium citrate $(\mathrm{pH} 6.0)$, followed by incubation with $0.3 \% \mathrm{H}_{2} \mathrm{O}_{2}$ to quench endogenous peroxidase activity. Slides were blocked in $10 \%$ rabbit serum, and were incubated with rabbit polyclonal anti-ECT2 antibody (dilution 1:50) for $12 \mathrm{~h}$ at $4^{\circ} \mathrm{C}$. After washing, the slides were incubated with an avidin-biotin-peroxidase system (Vectasin elite ABC kit, Vector Labs, Burlingame, CA). Finally, sections were exposed for 10-20 min to $0.01 \%$ 3,3-diaminobenzidine (DAB) (Sigma) and $0.01 \%$ hydrogen peroxide in PBS. A total of $\geq 500$ tumor cells were examined at an original magnification of $x 400$ by light microscopy, and intensity of positively stained cells was recorded for each immunostained specimen. At the time, observers were unaware of case numbers. ECT2 expression was evaluated according to an immunohistochemical scoring system depending on staining intensity ( 0 : none, 1 : weak, 2 : moderate, 3: strong), and weighted average (sum of point $\mathrm{x}$ area \%) of expression.

RNA isolation and quantitative PCR. Total RNA was isolated from frozen samples using Isogen (Nippongene, Toyama, Japan). Then, first-strand cDNA was amplified using oligo (dT) primers, and SuperScript II RNase H (Life Technologies, Grand Island, NY) according to the manufacturer's instructions. Quantitative PCR was performed with a LightCycler (Idaho Technology, Salt Lake City, UT). Each PCR reaction contained 1X LightCycler DNA Master SYBR Green I (Roche Molecular Biochemicals, Mannheim, Germany), $0.5 \mu \mathrm{M}$ of each primer, $3 \mathrm{mM} \mathrm{MgCl}_{2}$, and $2 \mu \mathrm{lcDNA}$ template. PCR conditions were: one cycle of denaturing at $95^{\circ} \mathrm{C}$ for $10 \mathrm{~min}$, followed by 40 cycles of $95^{\circ} \mathrm{C}$ for $15 \mathrm{sec}, 55^{\circ} \mathrm{C}$ for $5 \mathrm{sec}$, and $72^{\circ} \mathrm{C}$ for $10 \mathrm{sec}$. The 180-bp ECT2 PCR product was then subjected to a post-PCR melting cycle. Fluorescence intensity was calculated at each cycle, and a standard curve was constructed with 4-fold serial dilutions of cDNA obtained from glioma cell lines. Primer sequences for PCR amplification were: 
Table I. Patient characteristics.

\begin{tabular}{lrcc}
\hline Pathological diagnosis & $\begin{array}{c}\text { Number } \\
\text { of cases }\end{array}$ & $\begin{array}{c}\text { Gender } \\
\text { (male/female) }\end{array}$ & $\begin{array}{c}\text { Age } \\
\text { (mean } \pm \text { SD) }\end{array}$ \\
\hline Astrocytoma & 14 & $7 / 7$ & $37.6 \pm 17.9$ \\
Anaplastic astrocytoma & 9 & $3 / 6$ & $41.8 \pm 13.5$ \\
Glioblastoma & 31 & $20 / 11$ & $53.8 \pm 16.7$ \\
Total & 54 & $30 / 24$ & $47.6 \pm 17.8$ \\
\hline
\end{tabular}

ECT2 sense, 5'-CAA ATG GAT GCC CGA GCT G-3' and ECT2 antisense, 5'-GGT GGA AAT GGT GAC ACG TCT G-3' (Takara, Yotsukaichi, Japan).

siRNA treatment and cell proliferation assay. Specific siRNAs directed against human ECT2 were purchased from Invitrogen (Tokyo) for E053 and AMBION (Tokyo) for 26070. siRNAs were introduced into glioma cell lines by cytofectin-mediated transfection according to the manufacturer's instructions (Qiagen, Tokyo). For ECT2 RNA silencing, we tested two different siRNA oligonucleotide sequences. The sense and antisense sequences used were: 1) ECT2-HSS103053 (E053), 5'-AAA UCC UGU UCA UUC CGC CUU UCC C-3' (sense) and 5'-GGG AAA GGC GGA AUG AAC AGG AUU U-3' (antisense); 2) 26070, 5'-GGA CAU UAA AGU GGG CUU Utt-3' (sense) and 5'-AAA GCC CAC UUU AAU GUC Ctt$3^{\prime}$ (antisense). The negative control oligonucleotides were from Qiagen (Tokyo) with the following sequences: 5'-AGU ACU GCU UAC GAU ACG Gtt-3' (sense) and 5'-CCG UAU CGU AAG CAG UAG UAC Utt-3' (antisense). Cells were cultured in 96-well plates in $100 \mu 1$ serum-enriched medium. When $80 \%$ confluence was reached, $25 \mu 1100 \mathrm{nM}$ siRNA in cytofectin were added dropwise to the cell culture medium. Numbers of viable cells were evaluated after $48 \mathrm{~h}$ of culture by incubation with Tetra color one (Seikagaku Co., Tokyo), and values obtained were compared with those of controls. Controls involved the use of Cy3-labeled siRNA (Qiagen) directed against an unrelated mRNA (Luciferase; siRNA $\mathrm{LUC}_{\text {LC }}$ : Qiagen). Transfection efficiency was confirmed with Cy3-labeled siRNA $_{\text {LUC }}$ in each assay. All proliferation experiments were repeated as independent experiments at least 3 times. Results were expressed as means \pm standard deviation (SD) of three independent experiments.

Cell invasion of Matrigel. A transwell with an 8- $\mu \mathrm{m}$ diameter pore membrane (Becton-Dickinson, Tokyo, Japan) was coated with $500 \mu 1$ Matrigel (Becton-Dickinson) at $100 \mu \mathrm{g} / \mathrm{ml}$. Cells were left untreated, treated with control siRNA and with ECT2 siRNA, and transfected as described above. After 24-h incubation, cells were detached using cell dissociation solution (Sigma), washed twice with PBS, and resuspended in MEM containing $10 \%$ FBS. For the use of siRNA, a second transfection $24 \mathrm{~h}$ after the first was performed. In each case, $1 \times 10^{5}$ cells were seeded into the upper Matrigel-coated chamber of the Transwell. The lower chamber was filled with MEM supplemented with $10 \% \mathrm{FBS}$. After 24 -h incubation at $37^{\circ} \mathrm{C}$, the non-migrated cells in the upper chamber were gently detached by scraping, and adherent cells present on the lower
Table II. Correlation of immunohistochemical score and pathological diagnosis.

\begin{tabular}{lrrrr}
\hline & $0-1$ & $1-2$ & $2-3$ & Mean \pm SD \\
\hline Astrocytoma & 10 & 4 & 0 & $0.68 \pm 0.31$ \\
Anaplastic astrocytoma & 5 & 4 & 0 & $0.75 \pm 0.36$ \\
Glioblastoma & 11 & 17 & 3 & $1.18 \pm 0.58$ \\
\hline
\end{tabular}

surface of each insert were stained by Giemsa. Ten fields were counted by light microscopy at x200 magnification. Results were expressed against control values observed for incubation in untreated control for control and ECT2 siRNA.

Statistics. Differences in ECT2 expression between subgroups of gliomas were analyzed using the Mann-Whitney U test. Statistical significance was set at $\mathrm{P}<0.05$. Survival curves were estimated according to the method of Kaplan and Meier, and curves were compared using the generalized Wilcoxon's test. The log-rank test was used to assess the strength of association between survival time and single variables corresponding to factors thought to be prognostic for survival.

\section{Results}

Immunohistochemical analysis of ECT2 expression in glioma tissues. Immunohistochemistry was used to localize ECT2 proteins. Immunostaining patterns of glioma tissues are shown in Fig. 1. ECT2 expression was found in nuclei of tumor cells. We analyzed 54 available specimens, and correlations between ECT2 staining, patient survival, and histological grading were investigated. Glioblastoma patients (31 cases) showed strong staining compared to astrocytoma and anaplastic astrocytoma patients (23 cases) (Table II). When comparing between the high immunohistochemical score group and the low immunohistochemical score group, increased ECT2 expression inversely correlated with patient survival in glioblastoma plus anaplastic astrocytoma cases (log-rank test, $\mathrm{P}=0.031$; Fig. 2A), in glioblastoma cases (log-rank test, $\mathrm{P}=0.086$; Fig. 2B), and in all glioma cases (log-rank test, $\mathrm{P}=0.0074$; Fig. 2C).

Increased ECT2 gene expression in glioma inversely correlates with prognosis of patients. We compared expression levels of the ECT2 gene in 31 samples using quantitative real-time PCR. ECT2 gene expression was compared to histological grading. High expression of ECT2 mRNA was significantly associated with glioblastoma (GBM) compared to astrocytoma (A) (Fig. 3A; P<0.05). Patient survival curves, grouped according to levels of ECT2 gene expression in tumors, are shown in Fig. 3B. The high ECT2 expression group ( $\geq 0.023 \mathrm{pg} / \mathrm{ml}$ ) had significantly poorer prognosis than the low ECT2 expression group $(<0.023 \mathrm{pg} / \mathrm{ml})$ (log-rank test, $\mathrm{P}<0.05)$

Glioma cell proliferation and invasion are inhibited by ECT2 siRNA transfection. ECT2 overexpression was shown to be 

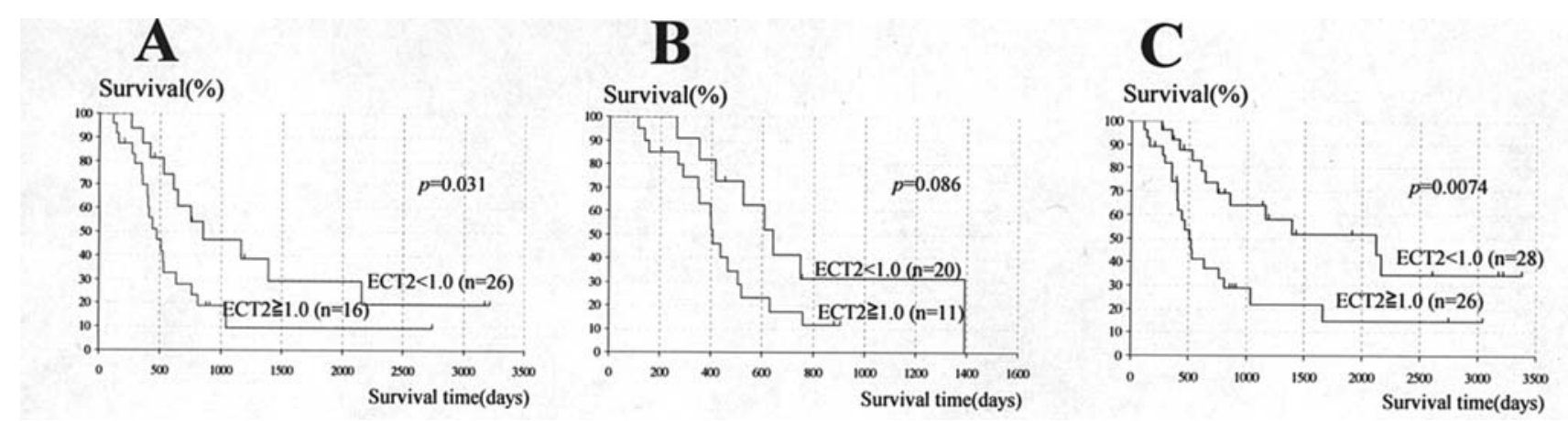

Figure 2. ECT2 protein expression and patient survival. Kaplan-Meier survival curves for patients who underwent surgical resection of glioma tissues were stratified according to ECT2 expression levels in the tumors. A comparison between protein expression (immunohistochemical score: sum of intensity $\mathrm{x}$ area \%) and overall survival of the glioma patients is shown. When the high immunohistochemical score group was compared to the low immunohistochemical score group, increased ECT2 expression correlated with patient survival in anaplastic astrocytoma plus glioblastoma cases (A; log-rank test, $\mathrm{P}=0.031)$, glioblastoma cases ( $\mathrm{B} ; \log$-rank test, $\mathrm{P}=0.086)$ and all glioma cases ( $\mathrm{C}$; log-rank test, $\mathrm{P}=0.0074)$.

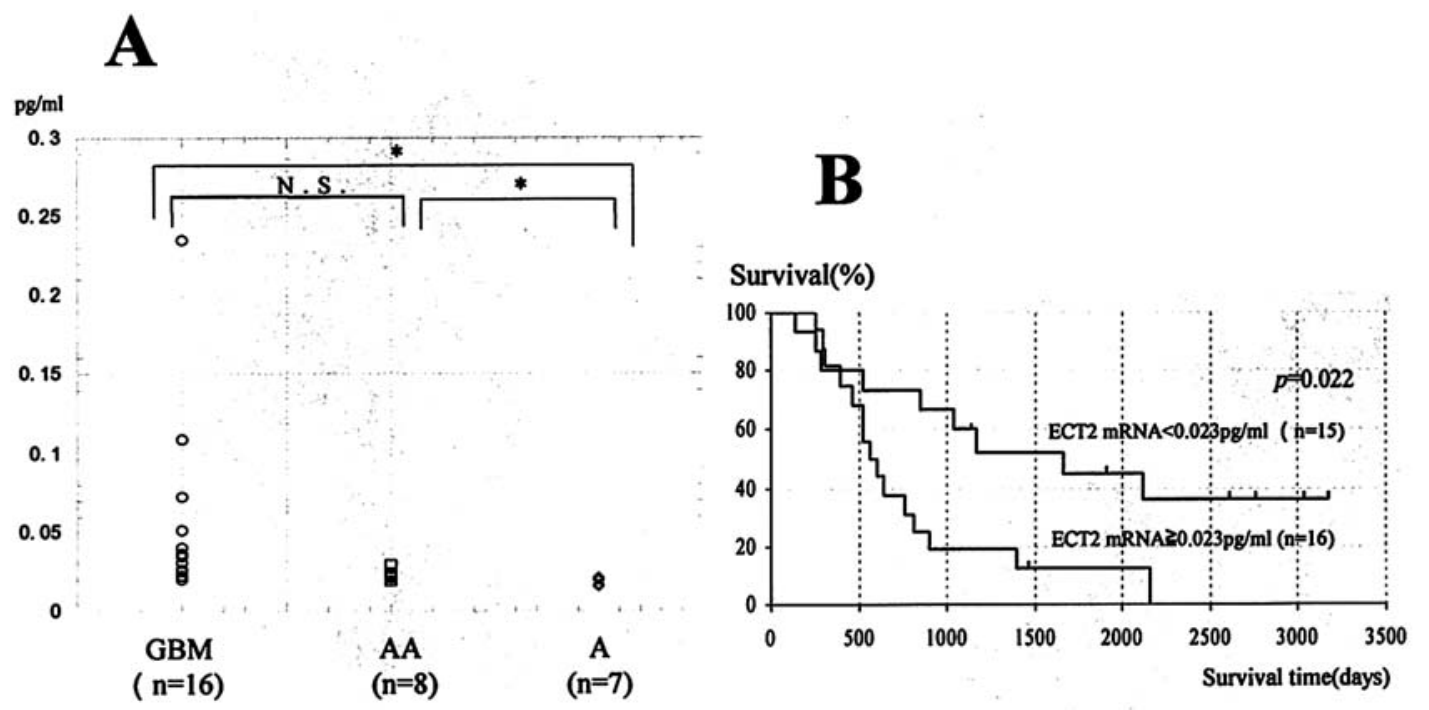

Figure 3. (A) ECT2 gene expression compared to histological grading. High expression of ECT2 mRNA was significantly associated with glioblastoma $(\mathrm{GBM})$ compared to astrocytoma (A) $(\mathrm{P}<0.05)$. N.S.: Not significant; ${ }^{*} \mathrm{P}<0.05$. (B) ECT2 mRNA expression and patient survival. Kaplan-Meier survival curves for patients who underwent surgical resection of glioma tissues were stratified according to expression level of ECT2 in tumors. Comparisons between mRNA expression and overall patient survival with gliomas (low ECT2 mRNA, 0-0.023 pg/ml; high ECT2 mRNA, $\geq 0.023$ pg/ ml; log-rank test, P=0.022).

linked with aggressiveness of glioma in our analysis. In order to determine whether down-regulation of endogenous ECT2 suppressed proliferation and invasive behavior of glioma, we synthesized siRNAs that, when transfected into cells, targeted ECT2 mRNA for degradation, thus reducing expression of ECT2 protein. We analyzed efficacy of siRNA-mediated inhibition of ECT2 synthesis in U251, GI-1, and T98G cells by real-time PCR. Since results obtained were virtually identical in these three cell types, only results from one cell line are presented here. As shown in Fig. 4A, transfection of T98G cells with ECT2 siRNA resulted in down-regulation of ECT2 mRNA $48 \mathrm{~h}$ after transfection. Finally, an unrelated control siRNA failed to modify ECT2 mRNA expression when transfected.

Following transfection with anti-ECT2 siRNA, T98G cell counts were suppressed by $\sim 40-50 \%$ in the following $48 \mathrm{~h}$, compared to untreated or control-siRNA-treated cells during the same period of time (Fig. 4B).
For invasion assays, transfectants were seeded onto Matrigel-coated invasion chambers, and were incubated for $24 \mathrm{~h}$. Then, the total number of cells on the underside of each filter was determined. As shown in Fig. 4C, transfection of T98G cells with ECT2 siRNA inhibited cell invasion through the Matrigel by $>50 \%$, whereas control siRNA had no effects. Invading cells were significantly suppressed by siRNA against ECT2, as reflected in the observed reduction of mRNA expression.

\section{Discussion}

Malignant gliomas are the most common primary brain tumors, and are generally considered as one of the deadliest of human cancers. Glioblastoma, the most pathologically aggressive form, shows a median survival time of just 9-14.6 months $(1,2)$. Advances in basic knowledge of cancer biology, as 


\section{A}

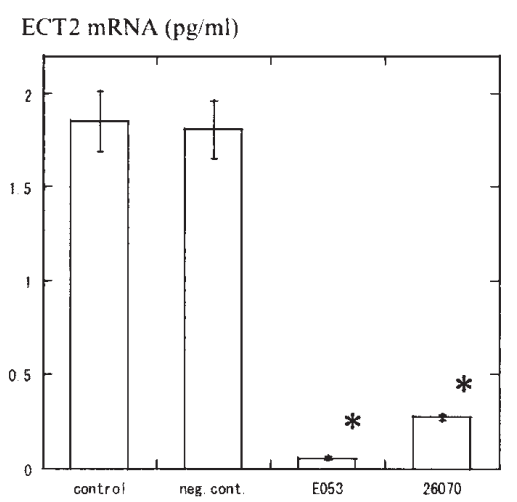

B

cell growth ratio(\%)

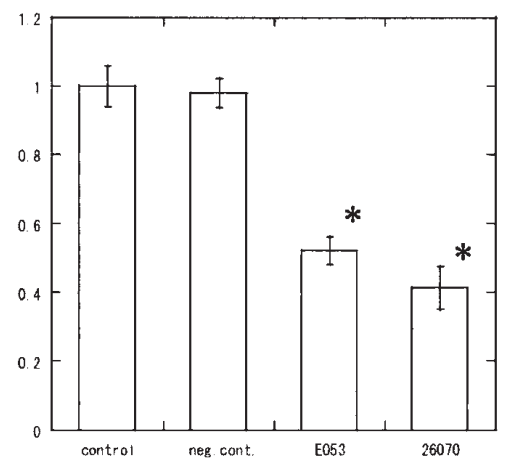

C

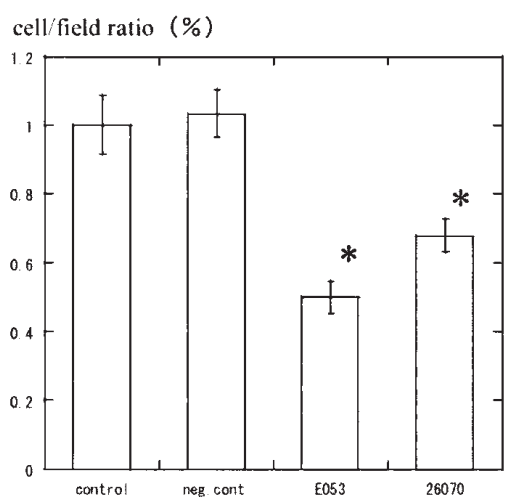

Figure 4. Effects of ECT2 knockdown by RNA interference on proliferation and invasiveness of human glioma cell lines. (A) Effects of an ECT2 siRNA on ECT2 mRNA expression in T98G glioma cell lines. After transfection with the ECT2 siRNA, ECT2 mRNA levels in the following $48 \mathrm{~h}$ were significantly suppressed compared to untreated or control siRNA-treated cells during the same time period. (B) Effects of ECT2 knockdown by RNA interference on proliferation of human glioma cells. T98G cells were transiently transfected with short interfering RNAs, and were subjected to a cell proliferation assay. Following transfection with ECT2 siRNA, T98G cell counts in the following $48 \mathrm{~h}$ were $\sim 40-50 \%$ of untreated or control-siRNA-treated cells during the same time period. (C) For the invasion assays, the transfectants were seeded onto Matrigel-coated invasion chambers, and were incubated for $24 \mathrm{~h}$. Total numbers of cells on the underside of each filter were determined. Invading cells were significantly suppressed by siRNA against ECT2, as reflected in the observed reduction of mRNA expression. Control: no siRNA treatment; neg. cont: control siRNA treated; E053, 26070: ECT2 siRNA treated; ${ }^{\mathrm{P}<0.01 .}$

well as surgical techniques, chemotherapy and radiotherapy, have led to only slight improvements in survival rates for glioblastoma (17). Therefore, there is a need to devise more effective therapeutic approaches, to better reveal the biological features of glioblastoma, and to discover a novel target molecule.

Reversi et al (18) first reported that genetic copy number gains on 3 q26.3 potentially involved the ECT2 gene in three glioblastoma cell lines, as seen by array comparative genomic hybridization. Yen et al (19) also reported copy number changes of ECT2 in esophageal squamous cell carcinoma. Overexpression of ECT2 increases its cytoplasmic localization, resulting in Rho activation leading to malignant transformation (5).

In this study, increased expression of ECT2 was associated with a malignant phenotype of glioma. In vitro, ECT2 siRNA inhibited glioma cell proliferation and invasion. These data suggest that increased expression of ECT2 might contribute to promotion of glioma invasiveness and progression.

No mutations in the ECT2 gene have been identified so far in a survey involving various clinical samples of cancers. Therefore, ECT2 most likely contributes to tumorigenesis through increased mRNA or protein expression as well as somatic or germline mutations.

On the basis of our data, ECT2 may play a role in the proliferation and invasion of glioma, and increased expression of ECT2 may contribute to the promotion of tumor invasiveness and progression. These data imply that evaluation of ECT2 expression is a prognostic marker for patients with glioma. Thus, ECT2 can be used as a novel molecular target for therapy, and as an important predictive marker for survival in glioma patients.

\section{Acknowledgements}

We are grateful to N. Kiyama and Y. Higuchi for their excellent technical assistance.

\section{References}

1. Stewart LA: Chemotherapy in adult high-grade glioma: a systematic review and meta-analysis of individual patient data from 12 randomized trials. Lancet 359: 1011-1018, 2002.

2. Stupp R, Mason WP, van den Bent MJ, Weller M, Fisher B, Taphoorn MJ, Belanger K, Brandes AA, Marosi C, Bogdahn U, Curschmann J, Janzer RC, Ludwin SK, Gorlia T, Allgeier A, Lacombe D, Cairncross JG, Eisenhauer E and Mirimanoff RO: Radiotherapy plus concomitant and adjuvant temozolomide for glioblastoma. N Engl J Med 352: 987-996, 2005.

3. Miki T, Smith CL, Long JE, Eva A and Fleming TP: Oncogene ect2 is related to regulators of small GTP-binding proteins Nature 362: 462-465, 1993.

4. Tatsumoto T, Xie X, Blumenthal R, Okamoto I and Miki T: Human ECT2 is an exchange factor for Rho GTPases, phosphorylated in G2/M phases, and involved in cytokinesis. J Cell Biol 147: 921-928, 1999.

5. Saito S, Liu XF, Kamijo K, Raziuddin R, Tatsumoto T, Okamoto I, Chen X, Lee CC, Lorenzi MV, Ohara N and Miki T: Deregulation and mislocalization of the cytokinesis regulator ECT2 activate the Rho signaling pathways leading to malignant transformation. J Biol Chem 279: 7169-7179, 2004.

6. Van Aelst L and D'Souza-Schorey C: Rho GTPases and signaling networks. Gene Dev 11: 2295-2322, 1997.

7. Hall A: Rho GTPases and the actin cytoskeleton. Science 279: 509-514, 1998

8. Etienne-Manneville $S$ and Hall A: Rho GTPases in cell biology. Nature 420: 629-635, 2002.

9. Hara T, Abe M, Inoue H, Yu LR, Veenstra TD, Kang YH, Lee KS and Miki T: Cytokinesis regulator ECT2 changes its conformation through phosphorylation at Thr-341 in G2/M phase. Oncogene 25: 566-578, 2006.

10. Niiya F, Tatsumoto T, Lee KS and Miki T: Phosphorylation of the cytokinesis regulator ECT2 at G2/M phase stimulates association of the mitotic kinase Plk1 and accumulation of GTPbound RhoA. Oncogene 25: 827-837, 2006.

11. Niiya F, Xie X, Lee KS, Inoue $H$ and Miki T: Inhibition of cyclin-dependent kinase 1 induces cytokinesis without chromosome segregation in an ECT2 and MgcRacGAP-dependent manner. J Biol Chem 280: 36502-36509, 2005

12. Prokopenko SN, Brumby A, O'Keefe L, Prior L, He Y, Saint R and Bellen HJ: A putative exchange factor for Rhol GTPase is required for initiation of cytokinesis in Drosophila. Genes Dev 13: 2301-2314, 1999

13. Somers WG and Saint R: A RhoGEF and Rho family GTPaseactivating protein complex links the contractile ring to cortical microtubules at the onset of cytokinesis. Dev Cell 4: 29-39, 2003. 
14. Kimura K, Tsuji T, Takada Y, Miki T and Narumiya S: Accumulation of GTP-bound RhoA during cytokinesis and a critical role of ECT2 in this accumulation. J Biol Chem 275: 17233-17236, 2000.

15. Echard A and O'Farrell PH: The degradation of two mitotic cyclins contributes to the timing of cytokinesis. Curr Biol 13: 373-383, 2003

16. Kleihues P and Cavenee WK: World Health Organization Classification of Tumours of the Nervous System. WHO/IARC Lyon, 2000.

17. Maher EA, Furnari FB, Bachoo RM, Rowitch DH, Louis DN, Cavenee WK and De Pinho RA: Malignant glioma: genetics and biology of a grave matter. Genes Dev 15: 1311-1333, 2001.
18. Roversi G, Pfundt R, Moroni RF, Magnani I, van Reijmersdal S Pollo B, Straatman H, Larizza L and Schoenmakers EF Identification of novel genomic markers related to progression to glioblastoma through genomic profiling of 25 primary glioma cell lines. Oncogene 2005.

19. Yen CC, Chen YJ, Pan CC, Lu KH, Chen PC, Hsia JY, Chen JT, Wu YC, Hsu WH, Wang LS, Huang MH, Huang BS, Hu CP Chen PM and Lin $\mathrm{CH}$ : Copy number changes of target genes in chromosome 3q25.3-qter of esophageal squamous cell carcinoma: TP63 is amplified in early carcinogenesis but downregulated as disease progressed. World J Gastroenterol 11: $1267-1272,2005$ 